\title{
Análisis de Empleabilidad e Industria 4.0 en el Ecuador, como Estrategia para Mejorar los Programas Educativos
}

\author{
Mgs. Marcel Oswaldo Méndez-Mantuano, \\ Mgs. Evelyn Carolina Egüez Caviedes, \\ Lic. Hillary Marissa Torres Ruiz, \\ Investigadores del Instituto Superior Tecnológico \\ Juan Bautista Aguirre, Ecuador
}

Ing. Mónica Alexandra Guilcapi Villacres,

Investigadora de la Universidad de las Fuerzas Armadas, Ecuador

Ing. Simón Bolivar Cruz Muñoz,

Investigador del Instituto Superior Tecnológico Simón Bolívar, Ecuador

Ing. Wilmer Xavier Oleas Vega,

Investigador de la Consultora Vera \& Asociados, Ecuador

Doi:10.19044/esj.2019.v15n34p44～URL:http://dx.doi.org/10.19044/esj.2019.v15n34p44

\section{Resumen}

En el Ecuador, existe el reto de rediseñar el sistema productivo y educativo, ya que en el primero, no se avizoran innovaciones tecnológicas significativas, mientras que la vigente oferta académica no sirve de soporte para la instauración de este tipo de diseños industriales. En este sentido, la presente investigación tiene como propósito analizar de manera global los niveles de empleabilidad y de automatización; posteriormente se detalla el panorama industrial del país y se culmina con la identificación de las propuestas educativas vigentes, donde se especifican aquellos programas que deben ser potenciados para garantizar la existencia de profesionales que respondan a las particularidades del mercado moderno. Para la determinación de los resultados, se analizaron reportes de instituciones que indagan en la relación de automatización y empleabilidad y se aplicó una encuesta para conocer la percepción de las personas sobre la posibilidad del reemplazo a corto plazo de los puestos de trabajo. Por ello, el enfoque aplicado en el estudio fue enfoque mixto, con un diseño documental y de campo, y se determinó un alcance explicativo. Finalmente se puntualizan estudios que establecen que la automatización no aumentará los niveles de desempleo, sino que el desarrollo de los procesos computarizados creará nuevas oportunidades laborales relacionadas a las actividades cognitivas no rutinarias, donde las posibles 
carreras del futuro estarán orientadas al desarrollo de las habilidades emocionales y exigirán un alto nivel de creatividad.

Palabras claves: Automatización, Educación, Empleabilidad, Industria 4.0, Robots

\title{
Employability and Industry 4.0 Analysis in Ecuador: A Strategy to Improve Educational Programs
}

\author{
Mgs. Marcel Oswaldo Méndez-Mantuano, \\ Mgs. Evelyn Carolina Egüez Caviedes, \\ Lic. Hillary Marissa Torres Ruiz, \\ Investigadores del Instituto Superior Tecnológico \\ Juan Bautista Aguirre, Ecuador \\ Ing. Mónica Alexandra Guilcapi Villacres, \\ Investigadora de la Universidad de las Fuerzas Armadas, Ecuador \\ Ing. Simón Bolívar Cruz Muñoz, \\ Investigador del Instituto Superior Tecnológico Simón Bolívar, Ecuador \\ Ing. Wilmer Xavier Oleas Vega, \\ Investigador de la Consultora Vera \& Asociados, Ecuador
}

\begin{abstract}
In Ecuador, there is a challenge of redesigning the educational system to become productive. First, this is because no significant technological innovations were envisioned, while the current academic offer does not support the establishment of this type of industrial design. This paper focuses on analyzing globally the levels of employability and automation. After then, the industrial landscape of the country was detailed and the identification of current educational proposals was also completed. This, however, specifies those programs that should be enhanced, to ensure the existence of professionals that respond to the particularities of the modern market. To determine the results, reports from institutions that investigate the relationship of automation and employability were analyzed. A survey was also applied to determine people's perception of the possibility of short-term replacement of jobs. A mixed approach was applied, with a documentary and field design, and an explanatory scope was determined. Finally, studies were performed which shows that automation will not increase unemployment levels, but that the
\end{abstract}


development of computerized processes will create new job opportunities related to non-routine cognitive activities. Here, possible future careers will be oriented towards the development of emotional skills and based on a high level of creativity.

Keywords: Automation, Education, Employability, Industry 4.0, Robots

\section{Introducción}

La cuarta revolución industrial podría impactar rápidamente una gran variedad de aspectos de la actual sociedad, rediseñando los paradigmas tecnológicos, económicos, sociales y educativos vigentes. Con seguridad, esta integración permitirá el desarrollo de avances científicos en campos como la robótica, inteligencia artificial, nanotecnología, computación cuántica, biotecnología, internet de las cosas, eficiencia energética, transportación, entre otros (Schwab, 2016).

La transformación industrial se basa en acelerados avances tecnológicos que inducirían cambios a nivel personal e industrial, permitiendo así una amplia y acelerada conexión interpersonal, mejorando categóricamente los aspectos relevantes de la vida diaria, así como procesos productivos de mayor eficiencia, los cuales incluyen la computarización de la manufactura principalmente, logrando notables avances en la reducción de los impactos negativos en el entorno natural (Barros, 2017).

Entre los efectos que ha traído la globalización económica se encuentra el traslado de las industrias a los países asiáticos, impactando la competitividad en países como Estados Unidos y los países del norte de Europa, que en el siglo pasado eran considerados como los motores industriales del mundo moderno. El desplome competitivo de estos países se puede vincular al efecto multiplicador natural del sector industrial. Sectores concatenados al mismo y el mercado en general, aún no recuperan los niveles de desempeño previo a la crisis del 2007, a pesar de que en los últimos años la industria de estos países ha mostrado una constante recuperación. La importancia del sector industrial radica en su capacidad de impulsar la innovación que eventualmente genera demanda de puestos de trabajos cualificados. En la Unión Europea, por cada euro producido por las industrias, 34 céntimos lo producen otros sectores no industriales. En eso radica la importancia de mantener elevados niveles de industrialización, por lo cual, los países que componen este bloque, proponen aumentar del 15,3\% al $20 \%$ el peso de la industria en el PIB para el año 2020 (Del Val, 2016).

Según la Organización de las Naciones Unidas para la Alimentación y la Agricultura (FAO), en el año 2011 los acuerdos comerciales entre Asia y América Latina ascendían a los 442.000 millones de dólares, con una mayor participación comercial proveniente de China, India, Japón y Corea del Sur. 
Estos intercambios no fueran posibles si los motores industriales como China no poseyeran altos porcentajes anuales de crecimiento, ocasionando la necesidad de materia prima para la elaboración de diferentes productos, y Latinoamérica es el lugar ideal para encontrar dichos productos indispensables para la industrialización (FAO, 2012). En el año 2017, las exportaciones a Asia crecieron el 20,1 \% frente al 18,1 \% del año 2016, y esta tendencia de crecimiento se mantendrá para los próximos años (CEPAL, 2017).

La automatización industrial intenta entonces revolucionar la actual matriz productiva, conjuntamente con mecanismos que garanticen la anhelada equidad social. Estas metas son casi utópicas en regiones tan desiguales como América Latina, en donde conviven teléfonos de última generación y un tercio de la población come una vez al día. Aunque estos cambios generalmente inducen transformaciones substanciales en la sociedad, en la actualidad no se han diseñado estrategias predictivas que permitan conjugar a las ciencias avanzadas con la conciencia social, con el objetivo de erigir un nuevo contrato social, y con ello lograr que la revolución tecnológica sea aliada en la reducción de la pobreza en el mundo. Con estas coyunturas, muchos plantean un nuevo paradigma productivo, donde unos suponen que la humanidad se enfrentaría con la tecnificación laboral y sus consecuencias en la empleabilidad obrera en varios aspectos industriales, mientras otros se identifican con lo mencionado por Chris Anderson: "Nosotros versus las máquinas es un equivocado esquema mental. Nos guste o no, todos (nosotros y nuestras máquinas) formamos parte de un inmenso cerebro conectado. Alguna vez tuvimos neuronas. Ahora, estamos siendo nosotros las neuronas", quien presupone que la humanidad no rivaliza con la robotización, sino que somos parte integral en esta simbiosis productiva (Beliz, 2017).

La automatización del empleo genera varias interrogantes en cuanto al aumento del desempleo de la población. En ese contexto, la Federación Internacional de Robótica (FIR), estimó que en el año 2017 funcionaban aproximadamente 1,3 millones de robots en las diferentes industrias en todo el mundo, divididos de manera preferencial en el sector automotriz, electrónico y metalúrgico, donde el $75 \%$ de estos se encuentran en países como Corea del Sur, Alemania y Japón. La misma FIR sostiene que se crean 4 empleos nuevos por cada empleo tecnológico, por ello, los países anteriormente citados tienen las más altas tasas de robots en el mundo con bajas tasas de desempleo entre la población económicamente activa (International Federation of Robotics, 2017).

En países como China el $77 \%$ de los trabajos puede automatizarse, los cuales no serán recuperados. Sin embargo, existen varias premisas positivas que determinan que la mecanización de las industrias mejorará la empleabilidad, ya que existirá una migración de las necesidades; es decir, las 
instituciones académicas deberán formar mano de obra especializada que opere las nuevas tecnologías en desarrollo (Beliz, 2017).

Los acuerdos comerciales entre los países asiáticos (principalmente) y latinoamericanos, han cimentado nuevas alianzas a nivel económico, político y social, las cuales han permitido que grandes inversiones se alojen en ambos subcontinentes, pero de manera preferencial en los países del sur de América. Esta interrelación puede ayudar a que los países latinoamericanos analicen las estrategias aplicadas en Asia para adaptarlas a las particularidades de cada país y con ello lograr altos niveles de productividad, automatización de los empleos e incremento de nuevas propuestas de empleabilidad.

La industria 4.0 es un nuevo modelo empresarial que se basa en el control de la cadena de valor a través de los ciclos de vida de los productos, apoyándose en las tecnologías de la información, con especial relevancia en el desarrollo de software. Esta etapa de metamorfosis industrial que vivimos en la actualidad consiste en mejorar y optimizar los procesos productivos de bienes y servicios a través de la implementación de robots, inteligencia artificial, computación en la nube, etc. Entre los temores que suscitan estos cambios acelerados, está la posible sustitución de la mano de obra humana por la autómata, lo que supondría una pérdida masiva de empleos en todo el mundo. Sin embargo, esta revolución industrial no está basada en el diseño de nuevas tecnologías emergentes, sino en la transición hacia nuevos sistemas que optimicen la infraestructura actual.

En este sentido, la presente investigación tiene como propósito analizar el impacto que trae consigo la denominada revolución industrial 4.0 (automatización de los procesos) en la empleabilidad del Ecuador, posteriormente se analizan las actuales ofertas educativas y finalmente se detalla una breve propuesta formativa que guarde consonancia con las tendencias productivas y de automatización industrial.

\section{Metodología}

El enfoque de la investigación es mixto, donde se detallan las características (cualitativamente) de la oferta educativa actual del país. Así mismo se indican valores porcentuales a través de gráficos sobre la empleabilidad y automatización de varios países del mundo y del Ecuador. El diseño es documental y de campo, ya que la información expuesta en esta investigación proviene de fuentes bibliográficas secundarias tales como artículos, informes técnicos, páginas de internet especializadas, entre otras. Se aplicó un análisis estadístico no probabilístico, por medio de una encuesta a 100 personas, para conocer la percepción sobre la factibilidad de reemplazo de los actuales trabajadores por autómatas (robots) en un lapso de 5, 10 y 15 años, donde la población objetivo fueron los ciudadanos mayores de 18 años que actualmente se encuentren laborando. Los resultados de la encuesta son 
una proyección de la población, la cual no representa a las empresas, ya que únicamente se deseaba conocer la percepción de las personas y no la perspectiva productiva. El alcance es explicativo, ya que se establecen las causales de la implementación de las industrias 4.0 y sus potenciales impactos en la empleabilidad y en el sistema de educación superior a nivel nacional.

\section{Desarrollo}

\section{Panorama general de la empleabilidad y la automatización}

Los niveles de creación de trabajo en los países asiáticos son notables, ya que los avances económicos han permitido mejorar los niveles de productividad en las últimas décadas. Sin embargo, existe la preocupación de que los mismos desaparezcan en los posteriores años, ya que las industrias optan por desarrollar sistemas productivos que potencien los resultados y minimicen los costos de producción.

En los últimos 25 años, en esta región se han creado alrededor de 30 millones de nuevos trabajos (no agrícolas) por año. Existe además un aumento gradual de la productividad y de los salarios, de tal forma que se ha reducido la pobreza, mejorando así la calidad de vida de las personas.

En la figura 1 se muestran los cambios de la tasa de pobreza en puntos frente al cambio estructural en porcentajes, y en la figura 2 se detallan los niveles de productividad alcanzados en los últimos 20 años frente al mismo cambio estructural anterior, en varios países asiáticos.

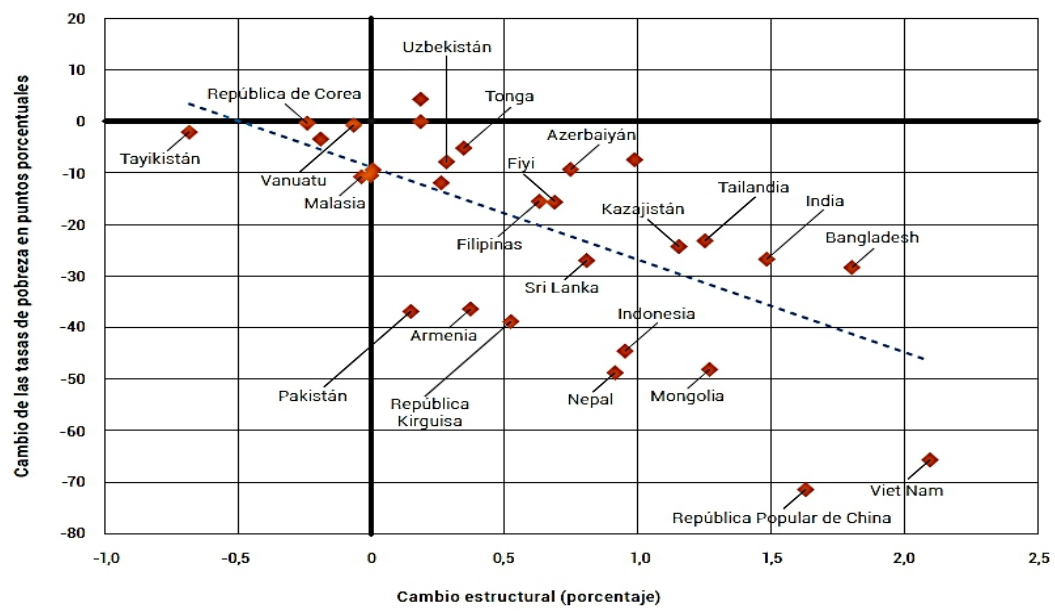

Figura 1. Reducción de la pobreza y componentes del crecimiento de la productividad, Asia en desarrollo, 19932013.

Fuente: African Development Bank Group, Asian Development Bank, Banco Interamericano de Desarrollo, European Bank for Reconstruction and Development, 2018 


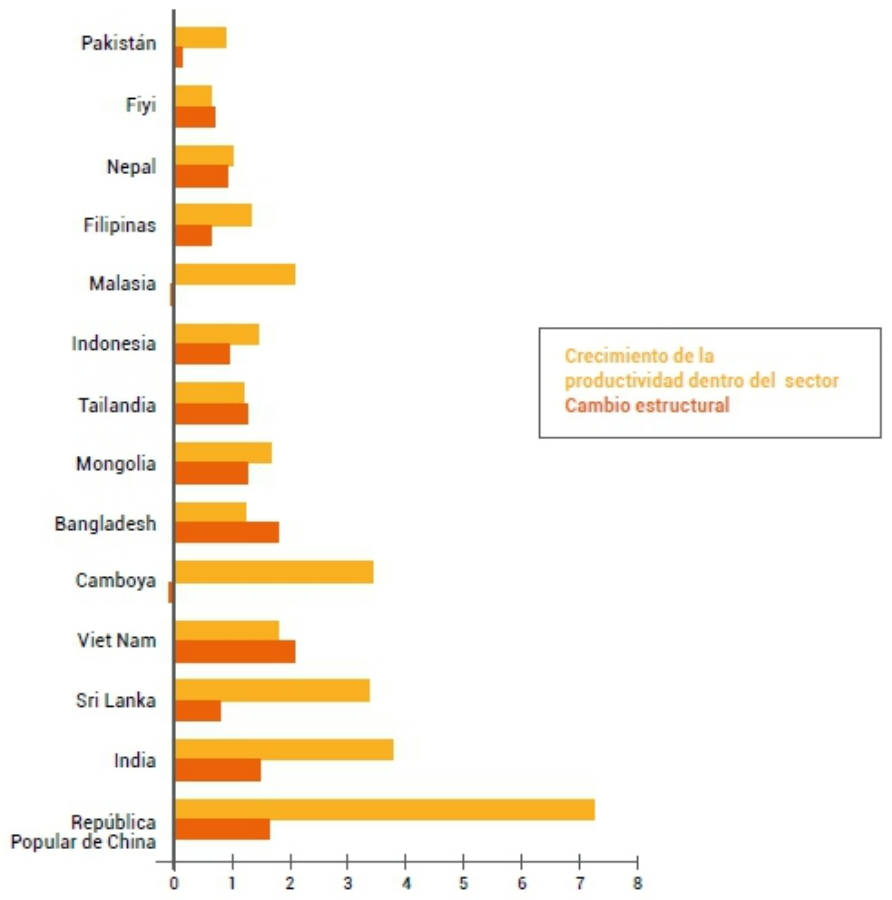

Figura 2. Componentes del crecimiento de la productividad, 1993-2013 (porcentaje).

Fuente: African Development Bank Group, Asian Development Bank, Banco Interamericano de Desarrollo, European Bank for Reconstruction and Development, 2018

Todo esto evidencia los notables desempeños económicos logrados en esta parte del mundo, ya que las efectivas inversiones en áreas tecnológicas y sociales (principalmente la educación), permitieron que la manufactura sea el motor de la producción en zonas que hasta no hace muchos años eran destinadas a la agricultura. No es menos cierto que la seguridad institucional de muchos países asiáticos permite un clima estable para las inversiones provenientes de Europa o América, en un contexto que favorece el crecimiento mutuo y sostenible (al menos en los últimos años).

En varios países del mundo (incluyendo los asiáticos), muchos robots industriales podrían ser programados para realizar tareas sin el acompañamiento de un humano, lo cual repercutiría de manera directa en la empleabilidad de las personas. En países como Vietnam, China, Indonesia, Filipinas, Bangladesh, India, Malasia y Sri Lanka, entre el año 2010 y 2015 existió un crecimiento de alrededor del $70 \%$ del número de maquinaria autómata en las industrias, equivalente a 887.400 unidades. Además, se ha evidenciado que existe un aumento en las tareas no rutinarias, las cuales están asociadas a trabajos en el ámbito tecnológico y de servicios no tradicionales, ergo las tasas de empleabilidad en tareas rutinarias se encuentran en 
contracción (African Development Bank Group, Asian Development Bank, Banco Interamericano de Desarrollo, European Bank for Reconstruction and Development, 2018).

Para el 2015, el área industrial automotriz acaparaba la mayor concentración de robots, con aproximadamente 40.000 unidades, seguidas por la metalurgia, productos plásticos y químicos, electrónicos y los textiles (Figura 3).

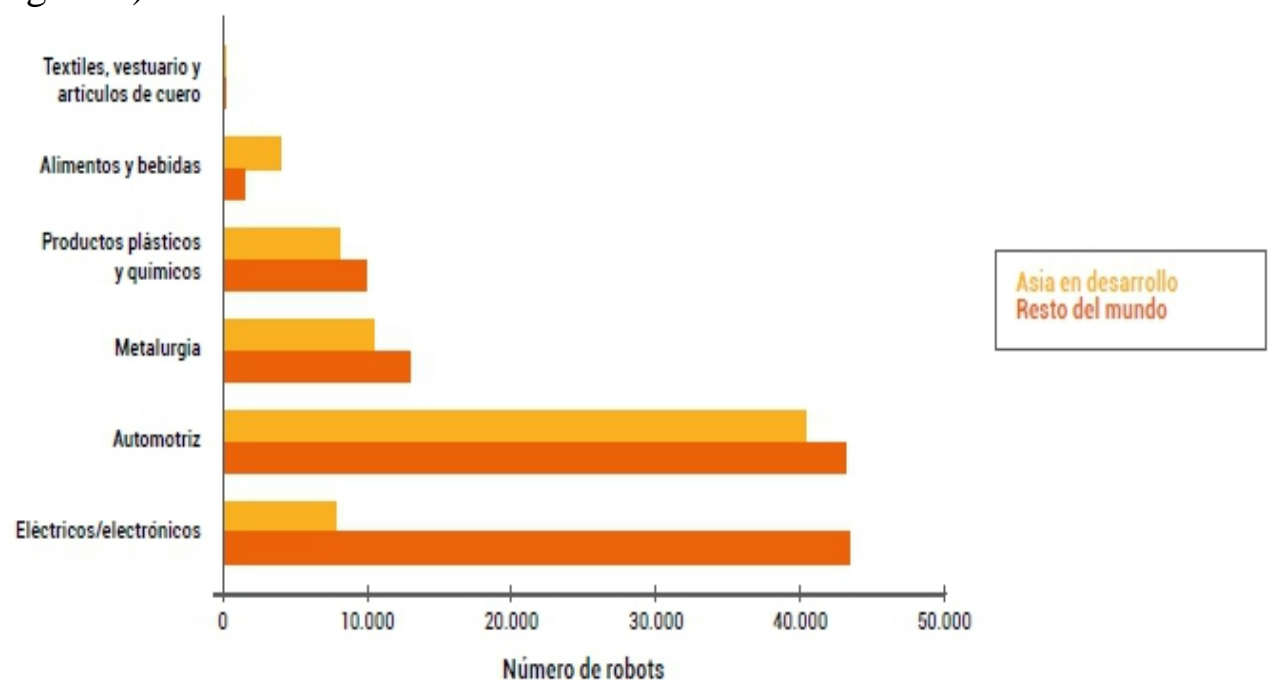

Figura 3. Robots por industria, Asia en desarrollo y el resto del mundo, 2015.

Fuente: African Development Bank Group, Asian Development Bank, Banco Interamericano de Desarrollo, European Bank for Reconstruction and Development, 2018

Por otro lado, la situación en América Latina ha ido evolucionando de manera promisoria en los últimos años (no a los niveles de los países del norte de América, de Europa o de Asia). Entre el año 2000 y 2017 los países de América Latina crecieron moderadamente al 2,7 \% en promedio, pero significativamente mayor a los países del OCDE y de la UE (African Development Bank Group, Asian Development Bank, Banco Interamericano de Desarrollo, European Bank for Reconstruction and Development, 2018).

Un tema pendiente para la región sigue siendo el aumento de los niveles de la productividad, los cuales se distancian de los países industrializados, y sin señales que se dé una cercana convergencia. Por ejemplo, en Estados Unidos el producto real por hora de trabajo es 2,5 veces mayor que en Chile (el país con mayor productividad de la zona), lo cual es preocupante, ya que se puede interpretar que un trabajador promedio es un 29 $\%$ el equivalente a un trabajador en los Estados Unidos (African Development Bank Group, Asian Development Bank, Banco Interamericano de Desarrollo, European Bank for Reconstruction and Development, 2018). 
Para el Banco Interamericano de Desarrollo, el $60 \%$ de los empleos en Latinoamérica corren el riesgo de perderse por la automatización; no obstante, esto no se dará a corto plazo, dado que los procesos de adaptación a nuevas tecnologías se han llevado de manera lenta en la mayoría de los países de la región, además de que no se cuenta con las capacidades, habilidades e infraestructura necesarias para dar cabida plena a esta revolución tecnológica. Como se muestra en la figura 4 , se estima que entre un $62 \%$ de los empleos en República Dominicana (el país con el menor grado de impacto) y un $75 \%$ en Guatemala (en el extremo contrario) podría desaparecer producto de la automatización (BID, 2018).

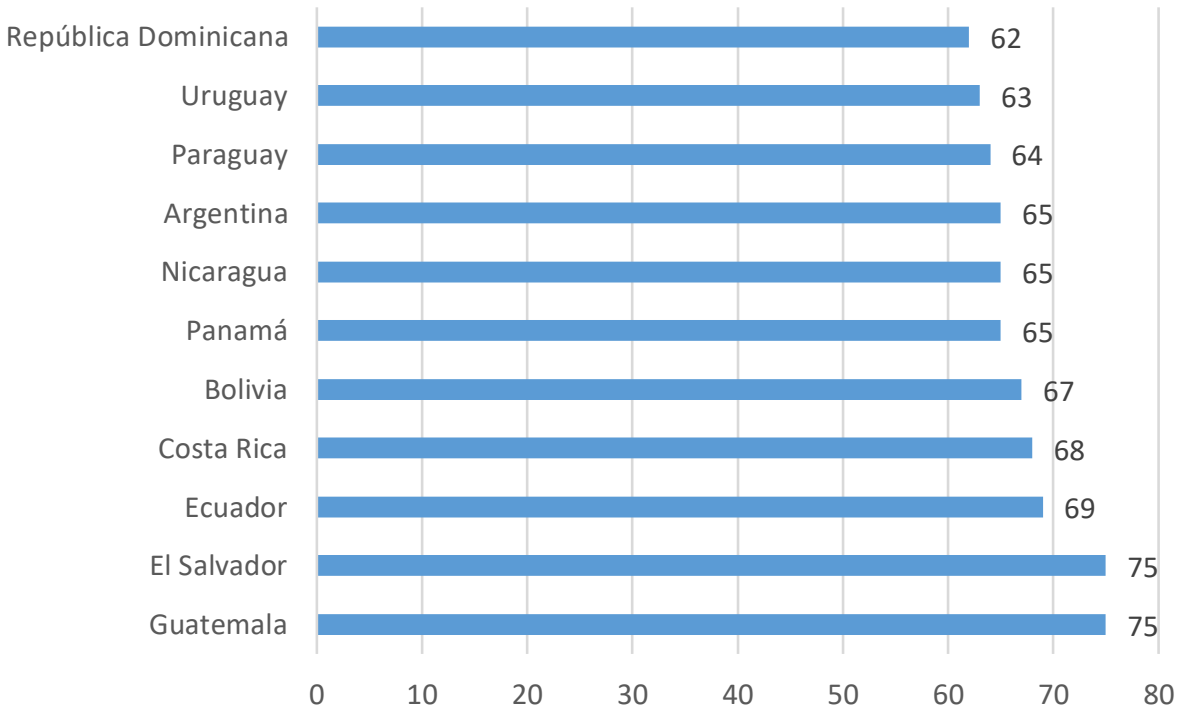

Figura 4. Porcentaje de trabajadores que se encuentran en ocupaciones con alto riesgo de automatización.

Fuente: BID, 2018

En el año 2013 los investigadores Frey y Osborne, determinaron la menor y mayor probabilidad de riesgo de desaparición de 702 tipos de empleos en los Estados Unidos, especificándose los siguientes en la tabla 1:

Tabla 1. Trabajos con menor probabilidad de desaparición en Estados Unidos. Fuente: Frey \& Osborne, 2013

\begin{tabular}{|c|c|c|}
\hline Ocupación & Ranking & Probabilidad \\
\hline Educadores sociales & 1 & 0,0028 \\
\hline $\begin{array}{c}\text { Supervisores de primera línea de } \\
\text { mecánicos, instaladores y reparadores }\end{array}$ & 2 & 0,003 \\
\hline Director de Gestión de Emergencias & 3 & 0,003 \\
\hline $\begin{array}{c}\text { Trabajadores de la salud mental y } \\
\text { adicciones }\end{array}$ & 4 & 0,0031 \\
\hline Otorrinolaringólogos & 5 & 0,0033 \\
\hline
\end{tabular}




\begin{tabular}{|c|c|c|}
\hline Acompañantes terapéuticos & 6 & 0,0035 \\
\hline Especialistas en prótesis & 7 & 0,0035 \\
\hline Cirujanos & 8 & 0,0036 \\
\hline $\begin{array}{c}\text { Primera línea de emergencias de incendios } \\
\text { y prevención }\end{array}$ & 9 & 0,0036 \\
\hline Nutricionistas & 10 & 0,0039 \\
\hline
\end{tabular}

Según la tabla 2, los trabajos con mayor probabilidad de desaparecer son:

Tabla 2. Trabajos con mayor probabilidad de desaparición en Estados Unidos.

Fuente: Frey \& Osborne, 2013

\begin{tabular}{|c|c|c|}
\hline Ocupación & Ranking & Probabilidad \\
\hline Gestión de reclamos & 689 & 0,98 \\
\hline Data Entry & 691 & 0,99 \\
\hline Bibliotecarios & 692 & 0,99 \\
\hline Agentes contables & 693 & 0,99 \\
\hline Trabajadores de procesamiento de imágenes y de máquinas & 694 & 0,99 \\
\hline Liquidador de impuestos & 695 & 0,99 \\
\hline Trabajadores de servicios de fletes & 696 & 0,99 \\
\hline Relojeros & 697 & 0,99 \\
\hline Proveedores de seguros & 698 & 0,99 \\
\hline Técnicos matemáticos & 699 & 0,99 \\
\hline
\end{tabular}

Tal como lo expresan los datos, la automatización se realizará en áreas donde las actividades son rutinarias, ya que el desarrollo de algoritmos para la Big Data consentirá establecer patrones de estos trabajos, de tal forma que las personas serán sustituidas por máquinas, las cuales bajarán los costos operativos de los procesos industriales o incluso administrativos.

\section{Empleabilidad y automatización en el Ecuador}

En el año 2019, existen aproximadamente 17’319.018 habitantes en Ecuador (proyección basada en el censo del 2010), cuya tasa de participación global en la economía se ubicó en el 66,8 \% (11'569.104 personas) y con un nivel de desempleo hasta junio que bordeó el 4,4\% (Instituto Nacional de Estadísticas y Censos, 2019). Al realizarse la proyección de la figura 4, aproximadamente 7.982 .682 empleos (69\% del total) se encuentran en un alto riesgo de ser reemplazados por robots.

En la figura 5, se muestran los niveles de empleo en el Ecuador por cada una de las ramas de las actividades económicas: 


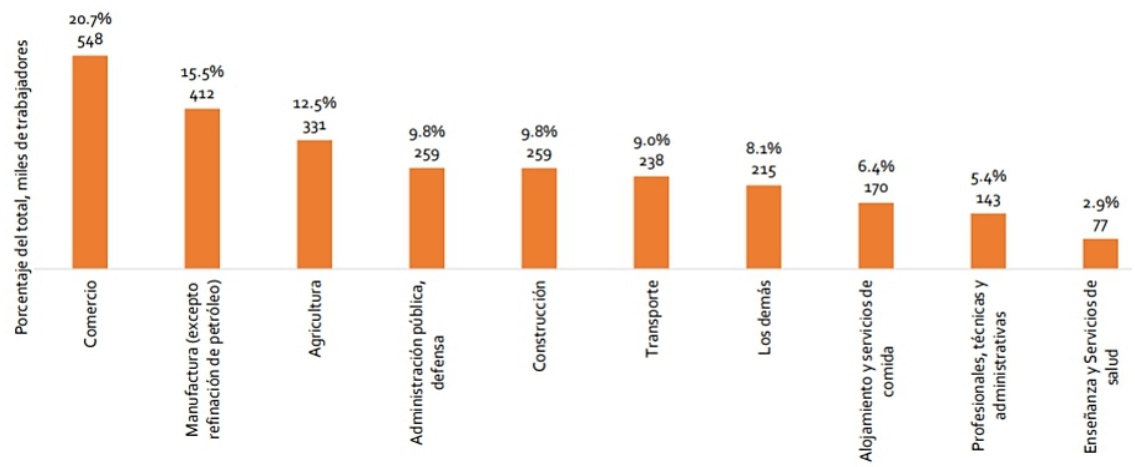

Figura 5. Trabajadores con empleo adecuado por rama de actividad económica. Fuente: Cámara de Comercio de Guayaquil, 2019

En los últimos años ha existido un crecimiento significativo de los productos no petroleros (ver figura 6), los cuales dinamizan a la economía y la creación de los nuevos empleos y de ofertas laborales asociadas a esta.

Exportaciones

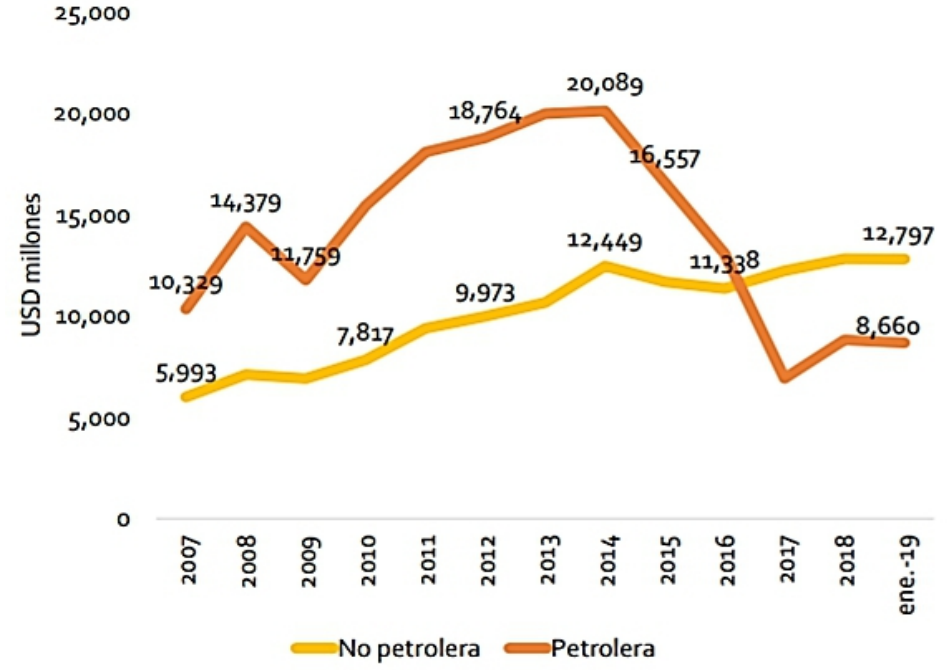

Figura 6. Evolución de las exportaciones no petroleras y petroleras desde el 2007 al 2019.

Fuente: Cámara de Comercio de Guayaquil, 2019

En el Ecuador no se han realizado estudios para determinar cuáles son los empleos más susceptibles a perderse por la automatización; no obstante, a nivel regional existen investigaciones que sirven como referencia para este análisis, ya que la mayoría de los países latinoamericanos comparten características similares en los aspectos culturales, sociales, demográficos, productivos, económicos, etc.

Aboal y Zunino (2017), realizaron un análisis de la probabilidad de automatización de las ocupaciones por las principales ramas de actividades 
económicas para los países de Argentina y Uruguay, teniendo los siguientes resultados en la tabla 3 :

Tabla 3. Probabilidad de automatización de las ocupaciones por rama de actividad.

Fuente: Aboal \& Zunino, 2017

\begin{tabular}{|c|c|c|}
\hline Rama de actividad & Argentina & Uruguay \\
\hline Actividades financieras & $67,00 \%$ & $79,00 \%$ \\
\hline Actividades inmobiliarias & $53,60 \%$ & $75,70 \%$ \\
\hline $\begin{array}{c}\text { Agricultura, Ganadería, Caza, } \\
\text { Forestación, Silvicultura y Pesca }\end{array}$ & $63,20 \%$ & $82,20 \%$ \\
\hline $\begin{array}{c}\text { Comercio al por mayor y por menor, } \\
\text { reparación }\end{array}$ & $76,60 \%$ & $80,00 \%$ \\
\hline Comunicaciones & $51,40 \%$ & $51,90 \%$ \\
\hline Construcción & $66,00 \%$ & $72,50 \%$ \\
\hline Electricidad, Gas y Agua & $69,30 \%$ & $67,00 \%$ \\
\hline Hoteles y Restaurantes & $66,60 \%$ & $76,30 \%$ \\
\hline Industria Manufacturera & $73,70 \%$ & $74,80 \%$ \\
\hline Minas y Canteras & $66,00 \%$ & $70,20 \%$ \\
\hline Otras actividades de servicio & $54,10 \%$ & $52,70 \%$ \\
\hline Transporte y Almacenamiento & $76,60 \%$ & $72,20 \%$ \\
\hline
\end{tabular}

Así mismo, estos autores analizaron la automatización y su probabilidad en los diferentes niveles educativos, presentando los siguientes datos relevantes que se muestran en la figura 7 :

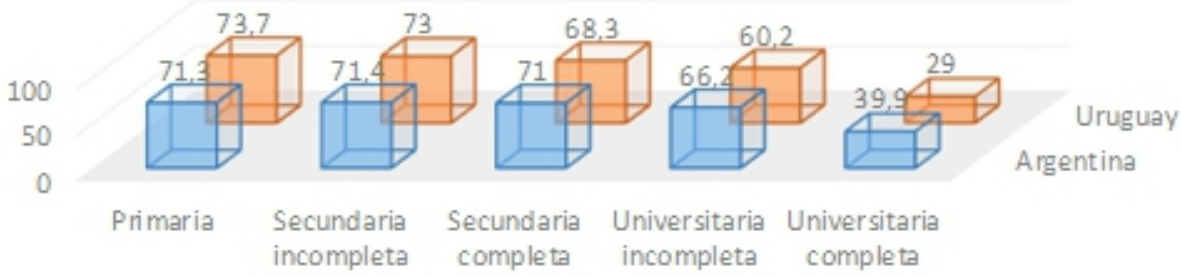

argentina Uuruguay

Figura 7. Probabilidad de automatización de las ocupaciones por nivel educativo.

Fuente: Aboal \& Zunino, 2017 
Tal como se presenta en la figura 8, se determinó el nivel hipotético de automatización por grupos de edades, con los siguientes datos porcentuales:

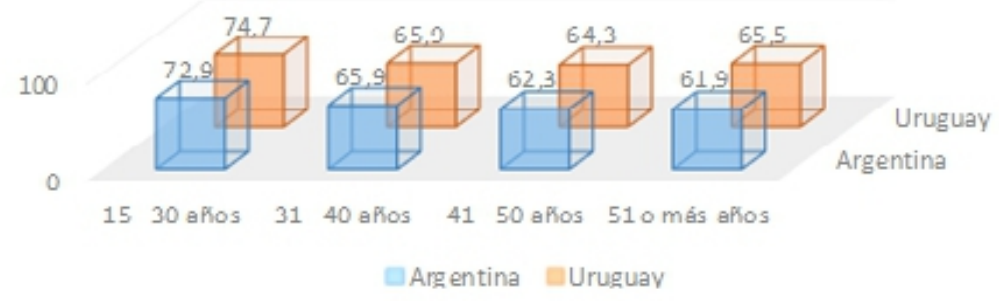

Figura 8. Probabilidad de automatización de las ocupaciones por grupos de edad. Fuente: Aboal \& Zunino, 2017

En los países de América Latina (incluido Ecuador), los niveles de automatización son lentos (en comparación con los países desarrollados), lo cual significaría que esta región se seguirá manteniendo relegada de los avances científicos, con implementaciones tardías de las nuevas tecnologías. Varios estudios han indagado sobre la relación directa entre implementación tecnológica y el desarrollo social y económico de una sociedad, y sus resultados comprueban que estos dos factores se encuentran interrelacionados y son correspondientes entre sí. En otras palabras, con los actuales paradigmas productivos y tecnológicos, los países que no logren articular de manera satisfactoria los avances tecnológicos como agentes de producción y de transformación de la misma sociedad, serán quienes mantengan los actuales niveles de pobreza y desigualdad.

La mayor conectividad y facilidad de acceso implican que la economía digital se encuentra en ascenso, lo que provoca el desarrollo de las infraestructuras necesarias para estas tecnologías. Además, se diseñan nuevas plataformas o softwares que permiten su ejecución y finalmente se crean servicios relacionados a la tecnología (no hubiera sido factible sin los dos elementos anteriores), lo que permite el dinamismo de actividades que en la actualidad únicamente las pueden realizar los humanos. Las tecnologías también son una oportunidad para los emprendedores, ya que sin ellas nunca se hubieran conformado empresas como Facebook, Google, Uber, Cabify, Airbnb, Alibaba, entre muchas otras, empresas que han dado respuestas al nuevo tipo de demandas de la sociedad.

Latinoamérica también ha dado su aporte para este nuevo mercado, pues en la actualidad las tecnolatinas (nombre con que se conocen las empresas de tecnología de América Latina y el Caribe) constituyen un ecosistema diverso con más de 5.000 empresas valoradas en $\$ 37.700$ millones de dólares (Surfing Tsunamis y Nxtp.Labs., 2017). 
Los índices de presencia de las Tecnologías de la Información y Comunicación en los hogares ecuatorianos de los años 2013 y 2017 son los que se exponen a continuación:

$\checkmark \quad$ En la figura 9 se detalla el porcentaje de equipamiento de los hogares (con edad de 5 años o más), lo que comprende al número de computadoras de escritorio, computadoras portátiles y ambas, en las familias ecuatorianas:

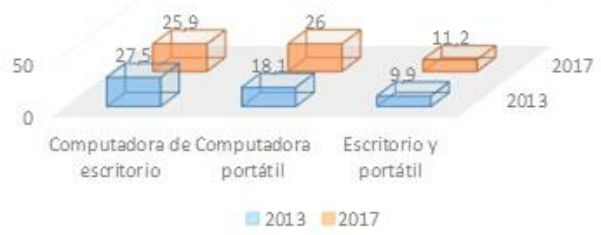

Figura 9. Porcentaje de hogares con computadoras.

Fuente: Instituto Nacional de Estadísticas y Censos, 2017

$\checkmark \quad$ Según la figura 10, los porcentajes de hogares (con edad de 5 años o más) que poseen teléfono fijo, celular y con acceso a internet, son los siguientes:

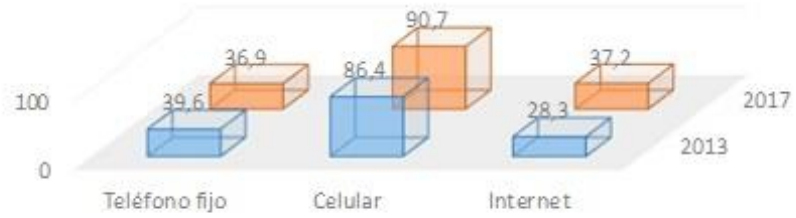

프를 2013

Figura 10. Porcentaje de hogares con teléfono fijo, celular y acceso de internet.

Fuente: Instituto Nacional de Estadísticas y Censos, 2017

$\checkmark \quad$ En la figura 11, se detalla el porcentaje de hogares (con edad de 5 años o más) que utilizan la computadora, el internet y que poseen teléfono inteligente, siendo los siguientes:

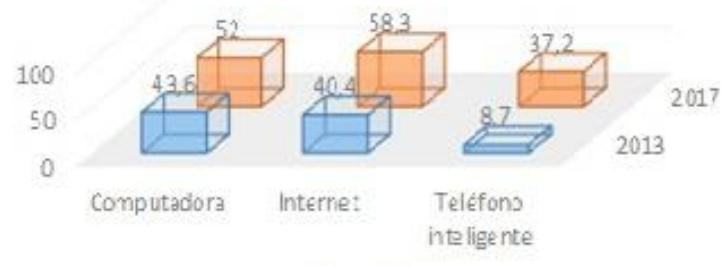

1020:3 $=2017$

Figura 11. Porcentaje de hogares que utilizan computadora, internet y que poseen teléfono inteligente.

Fuente: Instituto Nacional de Estadísticas y Censos, 2017 
En Uruguay el $67 \%$ de los hogares cuenta con un computador portátil, mientras que en Perú el $34 \%$ tiene uno, y en el Ecuador este índice está por el $25,9 \%$ (resultando uno de los porcentajes más bajos de la región). Por otro lado, el acceso a internet se encuentra menos extendido, donde Uruguay lidera con un $57 \%$, seguido por Chile con $53 \%$, Argentina con $52 \%$ y Brasil con $48 \%$. Por su parte, en Ecuador en el año 2017 el 37,2 \% de los hogares tenían internet. Aunque estos resultados no son significativamente altos (comparados con los países líderes), son mayores a los porcentajes de Bolivia, Nicaragua y Haití quienes se sitúan al final de la lista con valores que oscilan entre el 17\% y el $4 \%$. Se espera que para el 2020, en el $71 \%$ de los hogares latinoamericanos exista al menos un teléfono inteligente, y de cumplirse esta proporción sería mayor al promedio global equivalente al $66 \%$ (African Development Bank Group, Asian Development Bank, Banco Interamericano de Desarrollo, European Bank for Reconstruction and Development, 2018).

Ecuador ocupa el puesto 86 (de 140 puestos) en el ranking de competitividad global, cuya tendencia es a la baja, resultado que está muy por debajo de los países vecinos como Colombia y Perú, en los puestos 60 y 63 respectivamente (Financial Advisory \& Infrastructure, 2018).

En el país, las áreas en donde se han adoptado tecnologías emergentes relacionadas a la industria 4.0 (robots interconectados a internet), son aquellos sectores en donde se ensamblan autos y se fabrican tuberías, los cuales tienen mayor presión, con una funcionalidad del $100 \%$, y son capaces de garantizar la calidad dentro de todo el proceso productivo (bajando los costos operativos). Incluso en ciertas empresas se ha empezado a trabajar con aplicaciones de realidad virtual, simulaciones de los procesos industriales, impresiones 3D y el denominado internet de las cosas. Entre los resultados que se esperan está que los trabajos repetitivos o con bajo nivel de conocimiento vayan desapareciendo de a poco; sin embargo, trabajos con alto nivel de cualificación que demanden creatividad o un análisis a bases de criterios (no algorítmicos), serán cada vez más demandados por las industrias e instituciones.

Las anteriores perspectivas indican la baja adopción de la automatización en los procesos productivos, los cuales se alojan en la industria a pasos muy lentos; sin embargo, estos son constantes e irreversibles, por ello, ciertos sectores sociales han manifestado su preocupación por la pérdida de espacios laborales que serán sustituidos por robots de manera paulatina.

Es incierto poder determinar el tiempo en el cual se tendrá una implementación satisfactoria de este tipo de sistemas y procesos en las industrias ecuatorianas, ya que no existen estudios especializados que indiquen la cantidad de robots existentes, o en el que se detallen las áreas más susceptibles al reemplazo de humanos por robots, y de manera empírica es 
posible determinar que la sociedad en el país no siente un riesgo próximo ante esta eventualidad.

La premisa anterior es demostrada por los resultados obtenidos por la encuesta realizada a 100 personas en las ciudades de Daule y Guayaquil, en donde únicamente se preguntó si ellos consideraban factible que las empresas reemplacen a la mayoría del personal operativo en los próximos 5, 10 y 15 años, obteniéndose los siguientes resultados (Figura 12):

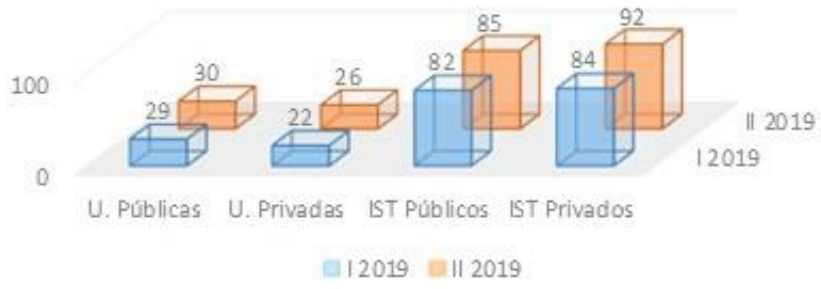

Figura 12. Factibilidad de reemplazo de las personas por robots en 5, 10 y 15 años

Aunque la mayoría de las personas no considera probable el reemplazo de la mano de obra humana por autómatas para los próximos años (5 y 10), esto no es un factor que impida esta tendencia a nivel nacional, ya que la evolución del sistema industrial no será interrumpida por ciertos sectores que desconocen las ventajas de modernizar los procesos. La industria 4.0 debe ser entendida por la sociedad como una oportunidad para disminuir la brecha tecnológica con relación a los países altamente industrializados, quienes han preferido diseñar nuevas estrategias que garantizan el acompañamiento humano en el ámbito industrial. En estos países se han estructurado nuevas ramas laborales, las cuales acompañan en la construcción de este nuevo sistema productivo. De igual manera presentan un alto nivel de cualificación de parte de las personas y por ende un mejor nivel social.

\section{Propuestas educativas en el Ecuador}

En los últimos dos semestres del 2019 (Figura 13), el número de instituciones de educación superior en el Ecuador que ofertaron carreras del tercer nivel eran las siguientes:

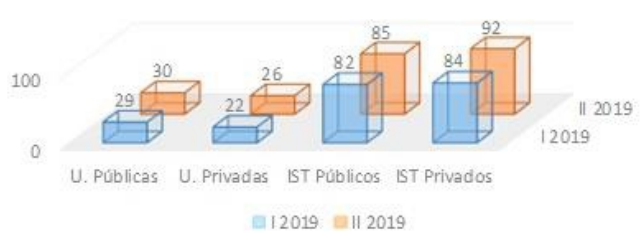

Figura 13. Número de instituciones de educación superior que ofertaron carreras en los dos semestres del 2019.

Fuente: Oferta Académica de las Instituciones de Educación Superior primer y segundo semestre del 2019 
Donde en el primer semestre se ofertaron 2.102 carreras, y en el segundo semestre 2.159 carreras.

En el primer semestre del 2018, las carreras más o menos demandadas por cada una de las principales actividades económicas del país fueron la que se detallan en la tabla 4:

Tabla 4. Detalle de las carreras más y menos postuladas en el primer semestre del 2018. Fuente: Oferta Académica de las Instituciones de Educación Superior primer y segundo semestre del 2019

\begin{tabular}{|c|c|c|c|}
\hline $\begin{array}{l}\text { Rama de la } \\
\text { actividad }\end{array}$ & $\begin{array}{c}\text { Participación } \\
\text { en el mercado } \\
\text { laboral }\end{array}$ & $\begin{array}{l}\text { Carreras más } \\
\text { postuladas }\end{array}$ & $\begin{array}{l}\text { Carreras menos } \\
\text { postuladas }\end{array}$ \\
\hline $\begin{array}{c}\text { Agricultura, } \\
\text { ganadería, caza, } \\
\text { silvicultura y pesca }\end{array}$ & $29,4 \%$ & Agronomía & $\begin{array}{c}\text { Acuicultura, Ingeniería } \\
\text { Agrícola, Ingeniería } \\
\text { Agrícola y Biológica }\end{array}$ \\
\hline Manufactura & $11,1 \%$ & $\begin{array}{l}\text { Ingeniería civil, } \\
\text { Arquitectura }\end{array}$ & $\begin{array}{c}\text { Materiales, Tecnología } \\
\text { Superior en } \\
\text { Automatización e } \\
\text { Instrumentación, } \\
\text { Polímeros }\end{array}$ \\
\hline $\begin{array}{c}\text { Servicios sociales y } \\
\text { de salud }\end{array}$ & $6,2 \%$ & $\begin{array}{l}\text { Medicina, } \\
\text { Enfermería, } \\
\text { Odontología }\end{array}$ & $\begin{array}{c}\text { Gestión de Transporte, } \\
\text { Tecnología en Seguridad } \\
\text { y Prevención de Riesgos } \\
\text { Laborales }\end{array}$ \\
\hline Enseñanza & $6,2 \%$ & $\begin{array}{l}\text { Educación Básica, } \\
\text { Educación Inicial }\end{array}$ & $\begin{array}{l}\text { Educación Intercultural } \\
\text { Bilingüe, Educación en } \\
\text { Ciencias Experimentales }\end{array}$ \\
\hline $\begin{array}{l}\text { Actividades } \\
\text { profesionales y } \\
\text { administrativas }\end{array}$ & $4,3 \%$ & $\begin{array}{c}\text { Administración de } \\
\text { empresas, Derecho, } \\
\text { Psicología, } \\
\text { Comunicación, } \\
\text { Economía, } \\
\text { Contabilidad y } \\
\text { Auditoria }\end{array}$ & $\begin{array}{l}\text { Agroecología, } \\
\text { Biocomercio, } \\
\text { Oceanografía, } \\
\text { Geociencias }\end{array}$ \\
\hline
\end{tabular}

En el primer semestre del 2018, la rama Agricultura, Ganadería, Caza, Silvicultura y Pesca concentró el 29,4\% de empleos a nivel nacional; no obstante, carreras como Bioeconomía, Ecosistemas, Acuicultura, Ingeniería Agrícola y Biológica, e Ingeniería en Ciencias del Agua registraron una baja postulación. Así mismo, Geociencias, Polímeros y Mantenimiento Industrial son altamente requeridas por las empresas en el área de manufactura; sin embargo, únicamente 17 personas optaron por estudiar este tipo de carreras. El $64 \%$ de los postulantes concentra su interés solo en 16 carreras (las 5 más postuladas son: Medicina, Administración de Empresas, Derecho, Psicología y Comunicación), pero la participación en el mercado laboral de estas carreras no sobrepasa el 4,3 \% (Secretaría de Educación Superior, Ciencia, Tecnología e Innovación, 2018). 
En la primera postulación del 2019 las tendencias no variaron de manera significativa, donde las siguientes carreras fueron las más y menos demandadas:

Carreras más postuladas en universidades públicas:

1. Administración de Empresas (13.819 postulantes)

2. Medicina (11.378 postulantes)

3. Enfermería (11.344 postulantes)

4. Derecho (8.586 postulantes)

5. Contabilidad y Auditoría (7.131 postulantes)

Carreras menos postuladas en universidades públicas:

1. Tecnología Superior en Mecánica Automotriz (0 postulantes)

2. Tecnología Superior en Automatización e Instrumentación (1 postulante)

3. Tecnología Superior en Logística y Transporte (2 postulantes)

4. Tecnología Superior en Seguridad y Prevención de Riesgos Laborales (3 postulantes)

5. Polímeros (7 postulantes)

Carreras más postuladas en Institutos Técnicos y Tecnológicos públicos:

1. Tecnología en Desarrollo Infantil Integral (1.447 postulantes)

2. Tecnología Superior en Mecánica Automotriz (1.247 postulantes)

3. Tecnología Superior en Marketing (1.108 postulantes)

4. Tecnología Superior en Desarrollo de Software (1.101 postulantes)

5. Tecnología Superior en Electricidad (1.033 postulantes)

Carreras menos postuladas en Institutos Técnicos y Tecnológicos públicos:

1. Escultura con nivel equivalente a Tecnología Superior (1 postulante)

2. Tecnología Superior en FloriFruticultura (4 postulantes)

3. Pintura con nivel equivalente a Tecnología Superior (5 postulantes)

4. Tecnología Superior en Química (5 postulantes)

5. Instrumentista (7 postulantes)

Los datos anteriormente mencionados indican claramente que en el Ecuador existen contradicciones en relación a la oferta de carreras por parte de las instituciones educativas y la participación de estas en el mercado laboral. Las carreras afines con Agricultura, Ganadería, Caza, Silvicultura y Pesca, en el primer semestre del 2018 tuvieron aproximadamente 4.300 postulantes, y ninguna de las propuestas educativas relacionadas con estas áreas se encuentra entre las 15 carreras más demandas a nivel nacional. Esto hace que existan espacios laborales que no son ocupados por técnicos o 
especialistas relacionados con la rama o actividad industrial, existiendo además plazas (en las ramas de servicio) con una elevada demanda de personas que desean ocuparlas.

Aunque la mayoría de las carreras de nivel superior en el Ecuador se encuentran en el área de servicios, existen algunas propuestas educativas que acompañarán a las empresas en la transición desde el actual nivel industrial a la industria 4.0, entre las que se destacan:

1. Desarrollo de Software

2. Diseño y Mantenimiento de Redes

3. Electrónica y Automatización

4. Física

5. Ingeniera Automotriz

6. Matemática Aplicada

7. Ingeniería de Materiales

8. Mecatrónica

9. Nanotecnología

10. Tecnologías de la Información

11. Tecnologías Geoespaciales

12. Telecomunicaciones

13. Telemática

A nivel educativo se denotan que existen pocas opciones de carreras que asistan a las empresas en la implementación de la industria 4.0, ya que las ofertas educativas están relacionadas a las necesidades productivas; es decir, la industria (mercado) es quien define los programas académicos que se proponen a la ciudadanía y, como fue mencionado anteriormente, la industria ecuatoriana no muestra signos claros que evidencien un salto hacia la "digitalización productiva" a través de la implementación de las nuevas tecnologías de la información. Por tal motivo, no existen carreras orientadas al desarrollo de estos procesos innovadores a nivel tecnológico.

Incluso con la instalación de superordenadores o de sistemas computarizados en la nube, la intuición humana, la capacidad de entendimiento, el razonamiento y la imaginación, siguen siendo factores relevantes para la sociedad (donde se incluye a la industria) y no serán reemplazados con facilidad por los sistemas robóticos o de inteligencia artificial. Por esta razón, existen propuestas en varios países del mundo para diseñar las "carreras profesionales del futuro", las cuales estarán orientadas a suplir la falta de razonamiento de los sistemas autómatas (robots, software, etc.). Entre las principales propuestas educativas (novedosas) para los próximos años se encuentran:

1. Imagineering (Ingeniera en Imaginación), el cual es un término acuñado por Walt Disney para identificar a un profesional con una alta capacidad creativa, donde transforma dichas ideas en estructuras 
materiales que tiene un impacto emocional en las personas que las usan, con el objetivo final de transformar la fantasía en una realidad (Yaiza, 2010).

2. En el fututo se requerirá de Expertos en Simulación, ya que las nuevas industrias desean conocer de manera predictiva el comportamiento de las personas en sus diferentes variables, utilizando modelos de replicación digital para mejorar los procesos. Desde el diseño de la maquinaria hasta el mantenimiento, un modelo digital puede ayudar a brindar consistencia, a realizar un mantenimiento más rápido y a obtener mejores operaciones (Instituto Universitario de Tecnología Aplicada IMF, 2018).

3. Los Expertos en Big Data (denominados científicos de datos), también serán solicitados por la industria 4.0, ya que se requieren expertos capaces de analizar y especificar una gran cantidad de información generada cada día a partir de máquinas, plantas y procesos conectados; además, estos profesionales deberán realizar propuestas de innovación para efectuar los cambios pertinentes que garanticen mejoras en los procesos productivos (Instituto Universitario de Tecnología Aplicada IMF, 2018).

4. La Mecatrónica y la Robótica tendrán una proyección superior en los próximos años, ya que estas ramas de la ingeniería crearán las articulaciones mecánicas necesarias (fábricas inteligentes), que respondan al nuevo paradigma productivo con procesos más rápidos y eficientes.

Según el Banco Mundial (2016), la transformación educativa tendrá las siguientes particularidades:

"La transformación del sistema educativo hacia alternativas con mayor flexibilidad y dinámica deberán priorizarse ante el cambio acelerado que imponen las nuevas tecnologías. El sistema educativo universitario por créditos es una de las opciones cuya aceptación es bastante generalizada en cada vez más universidades del mundo. Complementariamente se potenciará la educación en línea con una mayor escala y disponibilidad. Los MOOCs (Massive Open Online Courses) ofrecen cursos de nivel universitario sin la necesidad de completar un programa entero de estudios, siendo una herramienta muy importante en el futuro. De acuerdo a una encuesta llevada a cabo por el INET, las habilidades blandas que serán más demandadas hacia 2020 por las empresas de la Región Centro de Argentina incluyen el trabajo en equipo, el manejo de herramientas digitales y el conocimiento de normas y reglamentos; mientras que las habilidades duras incorporan el diseño, la informática y programación así como el control de calidad" (ADEC, 2017). 


\section{Conclusiones}

En base al análisis bibliográfico se puede determinar que los trabajos con mayores probabilidades de ser sustituidos son los que se realizan de manera rutinaria y aquellos con menor probabilidad de reemplazo son los que demandan creatividad o un análisis no algorítmico. Según la Federación Internacional de Robótica (FIR), se crean 4 empleos nuevos por cada empleo tecnológico. Esto se evidencia en los países con las tasas más altas de robots industriales, los cuales poseen bajas tasas de desempleo entre sus ciudadanos.

Por su parte en el Ecuador, el proceso de automatización industrial es lento y tardío, lo que supone que los actuales trabajadores no serán reemplazados por robots a corto o mediano plazo. Sin embargo, esta misma peculiaridad mantendrá al país relegado de las transformaciones tecnológicas, lo cual mantendrá los actuales niveles de desigualdad y pobreza.

El aumento de la automatización esbozará la migración de las actuales necesidades, incluyéndose las nuevas tendencias educativas; donde los empleos que requieran de habilidades emocionales, de creatividad, artísticas y trato interpersonal, serán menos susceptibles de ser automatizados y más demandados por las industrias. Estos empleados poseerán elevados niveles de cualificación profesional.

De acuerdo a la propuesta educativa presentada, para la implementación de la industria 4.0 en el Ecuador se necesitará de profesionales formados en las siguientes carreras profesionales: Desarrollo de Software, Diseño y Mantenimiento de Redes, Electrónica y Automatización, Física, Ingeniería Automotriz, Matemática Aplicada, Ingeniería de Materiales, Mecatrónica, Nanotecnología, Tecnologías de la Información, Tecnologías Geoespaciales, Telecomunicaciones, Telemática, entre otras.

Un adecuado conocimiento de las actuales tendencias de transformación industrial, social y tecnológica, permitirá a las sociedades una rápida adaptación a estos nuevos paradigmas que sin duda alguna son irreversibles. Por ello, es necesario el diseño de nuevas investigaciones que permitan conocer la situación actual de automatización en el país y cuáles son los puestos laborales susceptibles al reemplazo por robots.

\section{References:}

1. Aboal, D., \& Zunino, G. (2017). Innovación y Habilidades en América Latina. Obtenido de http://www.cinve.org.uy/novedad/articulo-dediego-aboal-y-gonzalo-zunino-publicado-en-revista-integracion-ycomercio-42-robotlucion-del-bid-intal/

2. ADEC (2017). Los empleos del futuro. El futuro de los empleos. Córdoba: Agencia para el Desarrollo.

3. African Development Bank Group, Asian Development Bank, Banco Interamericano de Desarrollo, European Bank for Reconstruction and 
Development. (2018). El futuro del trabajo: perspectivas regionales. Washington, DC: AfDB, ADB, BID, EBRD.

4. Barros, T. (2017). La industria 4.0: aplicaciones e implicaciones. Sevilla, España. Recuperado el 2019, de http://bibing.us.es/proyectos/abreproy/91146/fichero/La+Industria+4. $0+$ Aplicaciones+e+Implicaciones.pdf

5. Beliz, G. (2017). Un contrato social tecnológico para América Latina. En B. I. Desarrollo, Robotlución (págs. 12-23). Banco Interamericano de Desarrollo.

6. BID (2018). El futuro del trabajo en América Latina y el Caribe: ¿Una gran oportunidad para la región? (versión interactiva). Banco Interamericano de Desarrollo. doi:http://dx.doi.org/10.18235/0001339

7. Cámara de Comercio de Guayaquil (2019). Cifras Macroeconómicas del Ecuador. Obtenido de http://www.lacamara.org/website/wpcontent/uploads/2018/02/Entorno-economico-ccg.pdf

8. CEPAL (2017). Comisión Económica para America Latina y el Caribe. Obtenido de https://www.cepal.org/es/boletin-estadistico-decomercio-exterior-de-bienes-en-america-latina-y-el-caribe

9. Del Val, J. (2016). Industria 4.0: la transformación digital de la industria. Obtenido de Conferencia de directores y decanos de Ingeniería Informática: http://coddii.org/wpcontent/uploads/2016/10/Informe-CODDII-Industria-4.0.pdf

10. FAO (2012). Organización de las Naciones Unidas para la Almentación y la Agricultura. Obtenido de http://www.fao.org/inaction/agronoticias/detail/es/c/509887/

11. Financial Advisory \& Infrastructure (2018). Reporte Global de Competitividad 2018-2019. Obtenido de https://www2.deloitte.com/content/dam/Deloitte/cr/Documents/finan ce/docs/181017-cr-fas-Reporte-Global-de-Competitividad-WEF.pdf

12. Frey, C., \& Osborne, M. (2013). The future of employment: how susceptible are jobs to computerisation? Obtenido de https://www.oxfordmartin.ox.ac.uk/downloads/academic/The_Future _of_Employment.pdf

13. Instituto Nacional de Estadísticas y Censos (2017). Tecnologías de la Información y Comunicación. Obtenido de https://www.ecuadorencifras.gob.ec/documentos/webinec/Estadisticas_Sociales/TIC/2017/Tics\%202017_270718.pdf

14. Instituto Nacional de Estadísticas y Censos (2019). Encuesta Nacional de Empleo, Desempleo y Subempleo (ENEMDU), junio 2019. Quito: INEC.

15. Instituto Universitario de Tecnología Aplicada IMF (2018). La Industria 4.0 busca empleados: ¿Qué perfiles son los más 
demandados? Obtenido de https://iuta.education/noticias/la-industria4-0-busca-empleados-que-perfiles-son-los-mas-demandados/

16. International Federation Robotics (2017). IFR.org. Obtenido de https://ifr.org/img/office/IFR_The_Impact_of_Robots_on_Employme nt.pdf

17. Schwab, K. (2016). La cuarta revolución industrial. Barcelona, España: Debate.

18. Secretaría de Educación Superior, Ciencia, Tecnología e Innovación (2018). Carreras necesarias para el desarrollo del país y con alto nivel de empleabilidad tienen poca demanda. Obtenido de https://www.educacionsuperior.gob.ec/carreras-necesarias-para-eldesarrollo-del-pais-y-con-alto-nivel-de-empleabilidad-tienen-pocademanda/

19. Surfing Tsunamis y Nxtp.Labs. (2017). Tecnolatinas: América Latina en el tsunami de la tecnología. Washington, DC: Banco Interamericano de Desarrollo.

20. Yaiza, P. (26 de Septiembre de 2010). Ingenieros de la imaginación en un mundo de juguetes. El Mundo. Obtenido de https://www.elmundo.es/elmundo/2010/09/24/cultura/1285321418.ht $\mathrm{ml}$ 